\title{
Membrane-displayed somatostatin activates somatostatin receptor subtype-2 heterologously produced in Saccharomyces cerevisiae
}

\author{
Keisuke Hara, Tomohiro Shigemori, Kouichi Kuroda and Mitsuyoshi Ueda*
}

\begin{abstract}
The G-protein-coupled receptor (GPCR) superfamily, which includes somatostatin receptors (SSTRs), is one of the most important drug targets in the pharmaceutical industry. The yeast Saccharomyces cerevisiae is an attractive host for the ligand screening of human GPCRs. Here, we demonstrate the utility of the technology that was developed for displaying peptide ligands on yeast plasma membrane, termed "PepDisplay", which triggers signal transduction upon GPCR activation. A yeast strain that heterologously produced human somatostatin receptor subtype-2 (SSTR2) and chimeric Ga protein was constructed along with membrane-displayed somatostatin; somatostatin was displayed on the yeast plasma membrane by linking it to the anchoring domain of the glycosylphosphatidylinositol anchored plasma membrane protein Yps1p. We demonstrate that the somatostatin displayed on the plasma membrane successfully activated human SSTR2 in S. cerevisiae. The methodology presented here provides a new platform for identifying novel peptide ligands for both liganded and orphan mammalian GPCRs.
\end{abstract}

Keywords: Membrane-displayed ligand, PepDisplay, Yeast GPCR assay, Cyclic peptide, Somatostatin receptor subtype-2, Chimeric Ga protein

\section{Introduction}

G-protein-coupled receptors (GPCRs) are proteins that possess seven transmembrane domains and constitute one of the largest gene families in the human genome (Jacoby et al. 2006). More than $50 \%$ of all drugs, with annual worldwide sales exceeding $\$ 50$ billion, regulate GPCR functions, and approximately $30 \%$ of these drugs directly target GPCRs (Hopkins and Groom 2002; Xiao et al. 2008). Although the human genome contains 720-800 GPCRs (Siehler 2008), it is still less than 50 GPCRs that are the targets of commercially available drugs (Eglen et al. 2007). The development of a robust, reliable, and cost-effective functional screening method for both known and orphan GPCRs is a major focus of the pharmaceutical industry (Thomsen et al. 2005). Accordingly, it is evident that the market for the discovery and development of drugs targeting GPCRs will continue to grow in the coming decade.

\footnotetext{
* Correspondence: miueda@kais.kyoto-u.ac.jp

Division of Applied Life Sciences, Graduate School of Agriculture, Kyoto University, Sakyo-ku, Kyoto 606-8502, Japan
}

A GPCR assay that uses the baker's yeast Saccharomyces cerevisiae has been established as an experimental system for characterizing human receptor pharmacology and signal transduction mechanisms (Dowell and Brown 2002). Its attractive features are its simplicity with respect to genetic manipulation, economical propagation, easy maintenance of stably expressing cell lines, availability of an evolutionarily highly conserved signaling pathway, and presence of only two endogenous GPCRs that can be readily eliminated (Ladds et al. 2005). In spite of the major differences in G-proteins and membrane lipid composition between yeasts and mammalian cells, numerous cases of successful production of functional mammalian GPCRs in yeast have been documented (Xue et al. 2008). In addition, yeasts are attractive hosts for the high throughput screening of peptides or proteins from large combinatorial libraries (Chao et al. 2006; Li et al. 2007; Ueda 2004), with a high transformation efficiency of plasmids of up to $1-1.5 \times 10^{8}$ transformants $/ \mu \mathrm{g}$ achieved (Benatuil et al. 2010). Furthermore, homologous recombination (Ito et al. 2008), also known as gap-repair cloning, can be employed in yeast, which supports the 
reliable and cost-effective construction of random plasmid libraries.

The membrane-tethered peptide ligand is rather a new concept that was first documented by Fortin et al. (2009). In yeast, the display of heterologous peptides or proteins on cell wall was established by fusion with a part of cell wall proteins (Ueda and Tanaka 2000). However, it is considered that the displayed peptide ligand needs to localize within the range allowing the access to membrane-localized GPCRs. Therefore, we displayed the peptide ligand on the plasma membrane rather than cell wall, by using an anchoring domain of the glycosylphosphatidylinositol (GPI)-anchored plasma membrane protein, Yps1p. In the previous study, the $\alpha$-factor, which is a 13-amino acid residue-long peptide agonist of the yeast pheromone response pathway, was successfully displayed on the yeast plasma membrane, and signal activation was observed by employing a fluorescent reporter gene assay (Hara et al. 2012a). Extended application of this system to human GPCRs, which comprise one of the most important types of drug targets that are being pursued today, would be a valuable technology.

Somatostatin is a naturally occurring gastrointestinal hormone that regulates various endocrine and exocrine processes (Patel 1999). It is a cyclic tetradecapeptide with a disulfide bond between $\mathrm{Cys}^{3}$ and $\mathrm{Cys}^{14}$ residues. Cyclic peptides are promising scaffolds for peptide therapeutics because cyclic structures increase not only the physiological (serum) stability of peptides but also their affinity for binding partners owing to their conformational rigidity (Katoh et al. 2011). In fact, somatostatin receptor ligands, most of which possess cyclic structures, have been widely used as safe and effective treatments for pituitary and neuroendocrine tumors (Low 2004; Patel 1999; Weckbecker et al. 2003). The effects of somatostatin are transduced by its binding to plasma membrane-localized somatostatin receptors (SSTRs). SSTRs are encoded by five subtypes (SSTRx; $\mathrm{x}=1-5)$ and compose a subset of the GPCR superfamily (Jacoby et al. 2006).

In this study, the range of application of our technology for displaying peptide ligands on yeast plasma membranes, namely "PepDisplay", was extended to the activation of human GPCR that was heterologously produced in S. cerevisiae. The methodology presented in this study could be useful for identifying novel peptide ligands for both liganded and orphan mammalian GPCRs.

\section{Materials and methods}

\section{Strains and media}

The haploid yeast (S. cerevisiae) strain W303-1A (MATa, ade2-1, his3-11,15, leu2-3,112, trp1-1, ura3-1) (Thomas and Rothstein 1989), and other strains that were constructed specifically for our study, and their genotypes are listed in Table 1 . The yeast strains were cultivated aerobically in YPD medium $(1 \%[\mathrm{w} / \mathrm{v}]$ yeast extract, $2 \%$ $[\mathrm{w} / \mathrm{v}]$ peptone, and $2 \%[\mathrm{w} / \mathrm{v}]$ glucose), or in synthetic complete (SC) medium lacking histidine, adenine, leucine, and tryptophan (SC-HALW), containing $0.67 \%(\mathrm{w} / \mathrm{v})$ yeast nitrogen base without amino acids, $2 \%$ glucose, Multiple Drop-outs (DSCK292; ForMedium, Norfolk, UK), and appropriate amino acids. The assay medium was buffered at pH 7.2 with $50 \mathrm{mM}$ PIPES. Agar $(2 \%[\mathrm{w} / \mathrm{v}])$ was added to produce YPD and SC solid media. Escherichia coli DH5 $\alpha$ (Toyobo, Osaka, Japan) was used as the host for recombinant DNA manipulation and grown in Luria-Bertani medium $(1 \%[\mathrm{w} / \mathrm{v}]$ tryptone, $0.5 \%[\mathrm{w} / \mathrm{v}]$ yeast extract, and $0.5 \%[\mathrm{w} / \mathrm{v}]$ sodium chloride) containing $100 \mu \mathrm{g} \cdot \mathrm{mL}^{-1}$ ampicillin. After selection, a single colony was used to isolate the plasmid for transforming the yeast.

\section{Expression vectors}

PCR was performed using KOD-Plus-DNA polymerase (Toyobo).

Multistep construction of the SSTR2 expression plasmid, p412-SSTR2, was initiated by inserting an SacI$K p n I$ fragment of the GPD promoter and CYC1 terminator from p413-GPD (Mumberg et al. 1995) into SacI and KpnI sites in pRS412 (Brachmann et al. 1998) to create p412-GPD. The DNA fragment encoding human SSTR2 was amplified from a human brain cDNA library (B1234035; BioChain, Hayward, CA, USA) with primers 5'-CGCggatccAAAAAAATGGACATGGCGGA TGAGC- $3^{\prime}$ and $5^{\prime}$-TGCGgtcgacTCAGATACTGGTTT GGAGGTCTC-3'. This fragment was inserted into the BamHI and SalI sites of p412-GPD to create p412-SSTR2.

Table 1 List of yeast strains used and constructed in this study

\begin{tabular}{lll}
\hline Strain & Genotype & Reference \\
\hline W303-1A & MATa; ade2-1; his3-11,15; leu2-3,112; trp1-1; ura3-1 & (Thomas and Rothstein 1989) \\
\hline WH-3 & MATa; ade2-1; his3-11,15; leu2-3,112; trp1-1; ura3-1; sst2 4 ; far1 $\Delta$ & (Hara et al. 2012a) \\
\hline WH-7 & MATa; ade2-1; his3-11,15; leu2-3,112; trp1-1; ura3-1; sst2 4 ; far1 $\Delta ;$ ste2 4 & Present study \\
\hline WH-9 & MATa; ade2-1; his3-11,15; leu2-3,112; trp1-1; ura3-1; sst2 4 ; far1 $\Delta ;$ ste2 $\Delta ;$ gpa1 $\Delta ;$ p413-GPA1(Gi2) & Present study \\
\hline WSTH-9 & $\begin{array}{l}\text { MATa; ade2-1; his3-11,15; leu2-3,112; trp1-1; ura3-1; sst2 } \Delta ; \text { far1 } \Delta ; \text { ste2 } \Delta ; \text { gpa1 } \Delta ; \text { p413-GPA1(Gi2), p412-SSTR2, } \\
\text { PYEXfet }\end{array}$ & Present study \\
\hline
\end{tabular}


The chimeric Go-producing plasmid, p413-GPA1 (Gi2), was constructed as follows. The DNA fragment that consists of the GPA1 promoter and the GPA1 ORF was amplified from W303-1A genomic DNA using primers 5' - CGCgagctcATGTGCAT TAAAGCAGTAATGATAA GACG-3' and $5^{\prime}$-GCGctcgag TCAAAACAAACCACAA TC T T TAAGG T T T T GC TGGATGAT TAGATCGG-3’. This chimeric Go-encoding sequence was inserted into the SacI and XhoI sites of p413-CYC (Mumberg et al. 1995), from which the $S a c \mathrm{I}-\mathrm{XhoI}$ fragment of the CYC promoter was removed, to create p413-GPA1(Gi2).

Plasmids for producing membrane-displayed somatostatin with different linker lengths (Table 2) were constructed as follows. DNA fragments with different linker lengths were amplified from pYS0 (Hara et al. 2012a) using the following primers: $5^{\prime}$-GCGggatccGGTGGA TCTGATTACAAGGATGACGATGACAAG-3', where the underlined linker sequence varied depending on the linker length (namely, (GGTGGATCT) $\mathrm{nGGT}(\mathrm{n}=0,1$, $2,3,4,7,13$ ) for linker length $1,4,7,10,13,22,40$; (GGTGGATCT) $)_{\mathrm{n}}$ GGTGGA $(\mathrm{n}=0,1,2,3,6)$ for linker length 2, 5, 8, 11, 20; (GGTGGATCT) $)_{\mathrm{n}}(\mathrm{n}=1,2,3,4,5$, $7,10)$ for linker length $3,6,9,12,15,21,30)$, and $5^{\prime}$ TGGCCGAgtcgac TCA GATGAATGCAAAAAGAAGA GAAATTAATG-3'. The fragments were then inserted into the BamHI and SalI sites of pYS0, resulting in plasmids pYS1, pYS2, pYS3, pYS4, pYS5, pYS6, pYS7, pYS8, pYS9, pYS10, pYS11, pYS12, pYS13, pYS15, pYS20, pYS21, pYS22, pYS30, and pYS40. Next, a DNA fragment encoding the glucoamylase secretion signal and somatostatin was amplified from pULD1 (Kuroda et al. 2009) with primers $5^{\prime}$-CGGggtaccATGCAACT GTTCAAT T TGCCAT TG-3' and 5'-CGCggatccCCA CAGGAT G TGAAAGT C TTCCAGAAGAAATTCTTG CAGCCAGCGGCAGAAACGAGCAAAGAAAAG-3'. It was inserted into the KpnI and $B a m H I$ sites of the plasmids above. The resulting plasmids were designated pYS0-sst, pYS1-sst, pYS2-sst, pYS3-sst, pYS4-sst,

Table 2 Constructed plasmids for membrane display of somatostatin in this study

\begin{tabular}{|c|c|}
\hline Plasmid & Description \\
\hline $\begin{array}{l}\text { pYS }_{n} \text {-sst }(n=0,1,2,3,4,5, \\
6,7,8,9,10,11,12,13,15, \\
20,21,22,30,40)\end{array}$ & $\begin{array}{l}\text { Membrane display of somatostatin } \\
\text { with FLAG tag and various } \\
\text { linker lengths }\end{array}$ \\
\hline pYSO-nega & $\begin{array}{l}\text { Membrane display of FLAG tag } \\
\text { (same construct as pYSO-sst } \\
\text { but lack somatostatin sequence, } \\
\text { and used as a negative control) }\end{array}$ \\
\hline $\begin{array}{l}\text { pYS-notag3-sst, } \\
\text { pYS-notag20-sst, } \\
\text { pYS-notag38-sst }\end{array}$ & $\begin{array}{l}\text { Membrane display of somatostatin } \\
\text { without FLAG tag }\end{array}$ \\
\hline $\begin{array}{l}\text { pYS21-sst(W8A), } \\
\text { pYS21-sst(C14A) }\end{array}$ & $\begin{array}{l}\text { Membrane display of } \\
\text { alanine-substituted somatostatin } \\
\text { with FLAG tag }\end{array}$ \\
\hline
\end{tabular}

pYS5-sst, pYS6-sst, pYS7-sst, pYS8-sst, pYS9-sst, pYS10-sst, pYS11-sst, pYS12-sst, pYS13-sst, pYS15sst, pYS20-sst, pYS21-sst, pYS22-sst, pYS30-sst, and pYS40-sst, respectively. Additionally, the DNA fragment encoding the glucoamylase secretion signal sequence, but lacking the somatostatin sequence, was amplified similarly with primers $5^{\prime}$-CGGggtaccATG CAACTGTTCAATTTGCCATTG-3' and 5'-CGCggatc cACCGGCAGAAACGAGCAAAGAAAAGTAAG-3'. It was then inserted into the KpnI and BamHI sites in pYSO. The resulting plasmid was named pYSO-nega and was used as a negative control (Table 2).

Plasmids for producing membrane-displayed somatostatin without FLAG tag (Table 2) were constructed as follows. DNA fragments that encoded the linker region and the anchoring domain of Yps1p were amplified from W303-1A genomic DNA with primers either 5'GCGggatccACATCAAGTAAAAGAAATGTTGGTGAT C-3', 5' -GCGggatccGGCGGTAGTGGAGGCAGCGGAGG CTCGGGAGGTTCAGGAGGCTCGGGAGGTACATCAA GTAAAAGAAATGTTGGTGATC-3' or 5'-GCGggatccGG TGGATCTGGTGGATCTGGTGGATCTGGTGGATCTG GTGGATCTGGTGGATCTGGCGGTAGTGGAGGCAGC -3 , and 5'-TGGCCGAgtcgacTCAGATGAATGCAAAAA GAAGAGAAATTAATG-3'. The fragments were then inserted into the BamHI and SalI sites of pYSO-sst, resulting in plasmids pYS-notag3-sst, pYS-notag20-sst, and pYSnotag38-sst.

Plasmids for producing membrane-displayed somatostatin with an alanine substitution (Table 2) were constructed as follows. A DNA fragment encoding the glucoamylase secretion signal and the modified somatostatin was amplified from pULD1 with primers 5'-CGGggtaccATGCAACTGTTCAATTTGCCATTG-3' and either $5^{\prime}$-CGCggatccACCACAGGATGTGAAAGT CTTAGCGAAGAAATTCTTGCAGCCAGCGGCAGA AACGAGCAAAGAAAAG3 ${ }^{\prime}$ or $5^{\prime}$-CGC-ggatccACCA GCGGATGTGAAAGTCTTCCAGAAGAAATTCTTG CAGCCAGCGGCAGAAACGAGCAAAGAAAAG-3', and was inserted into the $K p n \mathrm{I}$ and $B a m \mathrm{HI}$ sites in pYS21, generating the plasmids pYS21-sst (W8A) and pYS21-sst(C14A), respectively.

\section{Construction of yeast strains}

All yeast transformation procedures were conducted with a Frozen-EZ Yeast Transformation-II kit (Zymo Research Corporation, Irvine, CA, USA). The yeast strains used and constructed in this study are listed in Table 1.

The WH-7 strain, which carried SST2, FAR1, and STE2 disruptions, was obtained using the 2-step procedure described by Gueldener et al. (2002) as follows. The STE2 of strain WH-3 (Hara et al. 2012a) was disrupted using the loxP-URA3-loxP cassette, which had been amplified from pUG72 (Gueldener et al. 2002) with the primers 
5'-GTTACTTAAAAATGCACCGTTAAGAACCATATCC AAGAATCAAAAcagctgaagcttcgtacgc- $3^{\prime}$ and $5^{\prime}$-ATACC GAAGGTCACGAAATTACTTTTTCAAAGCCGTAAAT TTTGAgcataggccactagtggatctg-3'. Next, Ura- derivatives of the transformants were selected on media containing 5fluoroorotic acid (Boeke et al. 1987). STE2 disruption at the targeted locus and removal of URA3 were verified by PCR using appropriate primers. GPA1 disruption was conducted after introduction of p413-GPA1(Gi2) into the WH-7 strain because the absence of the G $\alpha$ protein causes constitutive activity due to the existence of free G $\beta \gamma$ subunits (Guo et al. 2003). The GPA1 of WH-7 with p413-GPA1(Gi2) was disrupted using the KanMX4 cassette that had been amplified from the genomic DNA of strain BY4741 gpa1A (EUROSCARF, Frankfurt, Germany) with the primers 5'-ACAAGATCATAGGTGGA TAAAGCAAGCCG-3' ${ }^{\prime}$ and $5^{\prime}$-GCCTAGTAGATCTTGA TTCTTTGTCACCTC-3', to create strain WH-9. The WH-9 strain was transformed with p412-SSTR2 and a signal detection plasmid (pYEX-fet (Hara et al. 2012a)), resulting in the strain WSTH-9. The WSTH-9 strain was transformed with one of the plasmids for producing membrane-displayed somatostatin.

\section{Cultivation conditions}

To prepare starter cultures, a series of WSTH-9 strains with one of the membrane-displayed ligand plasmids was grown in $10 \mathrm{~mL}$ of SC-HALW medium ( $\mathrm{pH}$ 7.2) at $30^{\circ} \mathrm{C}$ overnight. The cultures used for assays were initiated by inoculating the starter culture into $10 \mathrm{~mL}$ SC-HALW medium to give an initial optical density (OD) of 0.05 at $600 \mathrm{~nm}$ using a VMax Kinetic ELISA Microplate Reader (Molecular Devices, Sunnyvale, CA, USA). These cultures were grown at $30^{\circ} \mathrm{C}$ on a rotary shaker $(250 \mathrm{rpm})$ until the $\mathrm{OD}_{600}$ reached $0.5-0.6$ corresponding to the late log phase.

For signal activation by exogenously added somatostatin, WSTH-9 strains (without the membranedisplayed ligand plasmid) were cocultured with serial dilutions of somatostatin, which was added at the start of the main culture. These cultures were also grown at $30^{\circ} \mathrm{C}$ on a rotary shaker $(250 \mathrm{rpm})$ until the $\mathrm{OD}_{600}$ reached $0.5-0.6$ corresponding to the late log phase.

\section{Plate assay}

Two-hundred microliters of the main cultures with $\mathrm{OD}_{600}$ ranging over $0.5-0.6$ were transferred to a $96-$ well plate (353072; BD Falcon, BD Biosciences, San Diego, CA, USA), and the fluorescence emitted was measured with a Fluoroskan Ascent Fluorometer (Labsystems OY, Helsinki, Finland). A filter pair with an excitation wavelength at $485 \mathrm{~nm}$ and emission wavelength at $527 \mathrm{~nm}$ was used to detect the fluorescence of enhanced green fluorescent protein (EGFP) produced in the yeast cells. The $\mathrm{OD}_{600}$ of each well of the sample plate was measured simultaneously. In order to normalize the number of cells in each well, the fluorescence value was divided by the $\mathrm{OD}_{600}$ reading. The fluorescence of the negative control (pYS0-nega strain, see subsection on Expression vectors) was subtracted from those for all of the other samples.

\section{Western blot analysis}

A cell membrane fraction was prepared according to the previously described method (Frieman and Cormack 2004; Hara et al. 2012a). Membrane proteins were analyzed by electrophoresis on $15 \%$ (w/v) SDS polyacrylamide gels and electrotransferred onto a nitrocellulose membrane (Bio-Rad, Richmond, CA, USA). The membrane was pretreated with $5 \%(\mathrm{w} / \mathrm{v})$ skim milk powder in PBS (pH 7.4), overnight, for preventing the nonspecific binding of antibody. The membrane was incubated for $1 \mathrm{~h}$ at room temperature with mouse anti-FLAG M2-Peroxidase antibody (Sigma-Aldrich, St Louis, MO, USA) at a concentration of 1:10000 in 5\% skim milk/PBS. The blots were washed 5 times in 2\% (v/v) Tween/PBS for $5 \mathrm{~min}$, and antibody binding was detected using ECL-Plus (GE Healthcare, Buckinghamshire, UK).

\section{Results}

An overview of this study is illustrated in Figure 1. To optimize the signal output, gene deletions were performed on the negative regulator of Gpa1 activity (sst2 $\Delta$ ) and a cyclin-dependent kinase inhibitor $(\operatorname{far} 1 \Delta)$. For the production of human SSTR2 and chimeric Go subunit, gene deletions were performed on yeast endogenous GPCR (ste $2 \Delta$ ) and G-protein $\alpha$-subunit (gpa14) accompanied by the introduction of human SSTR2 expression plasmid and chimeric $G \alpha$ expression plasmid (Brown et al. 2000; Hara et al. 2012b). The chimeric Go subunit employed in this study was the "transplant" type, in which the C-terminal five amino acids of Gpalp (KIGII) were exchanged with the mammalian Gi sequence (DCGLF). Signal activation was detected by fluorescence from pYEX-fet (Hara et al. 2012a), which is the high copy number reporter plasmid that contains EGFP and is controlled by the signal responsive FUS1 promoter. When the constructed WSTH-9 strain (Table 1) was exposed to exogenously added somatostatin, signal activation was observed in a dose-dependent manner (Figure 2), indicating that the chimeric $G \alpha$ and human SSTR2 that were heterologously produced in the yeast were functional.

We introduced plasmids for the membrane display of somatostatin with different linker lengths into the WSTH-9 strain. The fluorescence intensity and $\mathrm{OD}_{600}$ of the cell culture were measured at the late log phase, and the fluorescence intensities of each strain divided by 


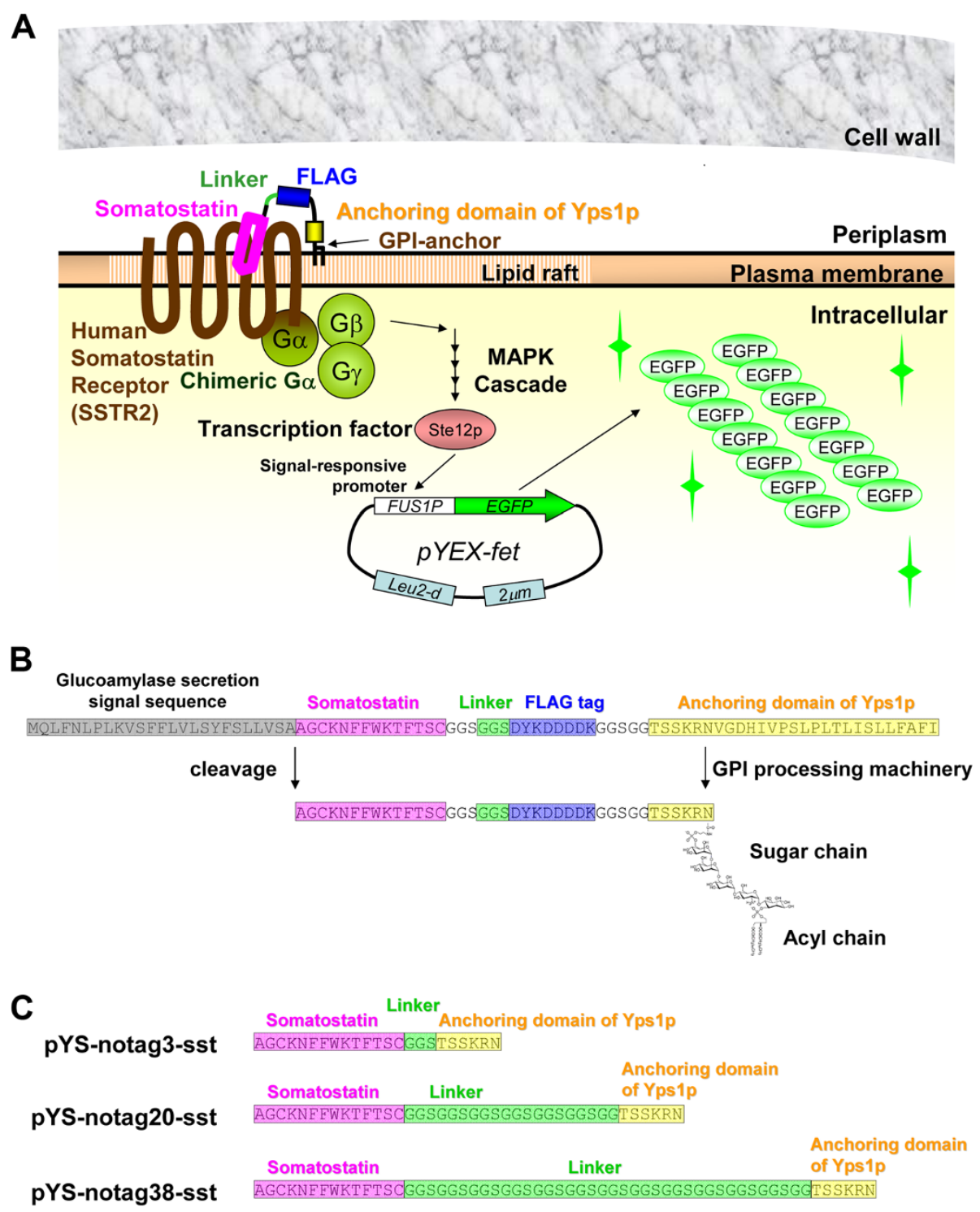

Figure 1 Schematic representation of signal activation of human somatostatin receptor subtype-2 by membrane-displayed somatostatin. A. Overview of this study. Membrane-displayed somatostatin (cyclic peptide) activates human somatostatin receptor subtype-2, which is heterologously produced in yeast, and leads to the activation of chimeric Ga proteins, the mitogen-activated protein kinase cascade, and transcription factor Ste12p. Phosphorylated Ste12p induces the overexpression of enhanced green fluorescent protein by binding to a pheromone response element in the FUS1 promoter of pYEX-fet (signal detection plasmid) (Hara et al. 2012a). This expression allows monitoring and quantification of signal transduction by fluorescence. B. Functional domains encoded by the membrane-displayed somatostatin plasmid. After processing by the secretory pathway, the signal sequence and glycosylphosphatidylinositol (GPI) targeting sequence are cleaved, and the somatostatin sequence, with a free N-terminus is displayed on the plasma membrane by GPI covalently linked to the C-terminus. C. Amino-acid sequence of membrane-displayed somatostatin without FLAG tag.

the $\mathrm{OD}_{600}$ were compared (Figure 3A). Among the broad spectrum of activities in a series of yeast strains that possessed one of the membrane-displayed somatostatin plasmids, a strain with pYS21-sst exhibited the strongest signal (Figure 3A). In addition, the production of membrane-displayed somatostatin with various linker lengths on the yeast plasma membrane was confirmed by western blot analysis of the membrane fraction using anti-FLAG tag antibody (Figure 3B). These results indicate that the membrane-displayed somatostatin directly activated the human SSTR2.

To confirm whether the signal activation triggered by the membrane-displayed somatostatin was due to the displayed somatostatin, alanine substitution into somatostatin was employed (Figure $3 \mathrm{C}$ ). $\operatorname{Trp}^{8}$ is critically important for agonistic activity and alanine substitution at $\operatorname{Trp}^{8}$ causes a significant loss in activity (Rosenthal et al. 1983; Vale et al. 1978). When $\operatorname{Trp}^{8}$ of 


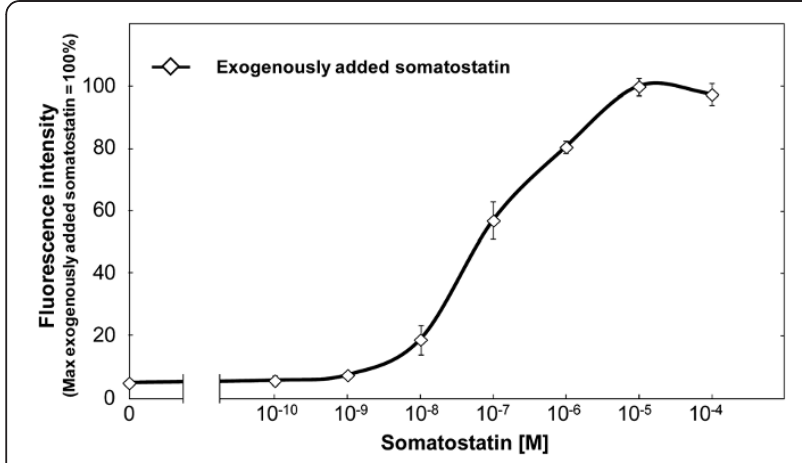

Figure 2 Activation of human somatostatin receptor subtype-2 produced in yeast by exogenously added somatostatin. Human somatostatin receptor subtype-2 was produced in yeast and stimulated by exogenously added somatostatin at various concentrations. The fluorescence intensity derived from receptor activation induced by enhanced green fluorescent protein was measured by using a 96-well fluorescent plate reader. Each data point represents the data (mean \pm standard error of the mean) of three independent experiments.

the membrane-displayed somatostatin (pYS21-sst) was substituted by alanine, the activity was significantly decreased (Figure 3C). This indicates that the activation was triggered by the somatostatin that was displayed on plasma membrane. Somatostatin is a naturally occurring cyclic peptide hormone with a disulfide bond between $\mathrm{Cys}^{3}$ and $\mathrm{Cys}^{14}$ residues. It has been reported that the hormonal activity was lost in a linearized form of somatostatin (Vale et al. 1978). To confirm that the membrane-displayed somatostatin retained its cyclic structure, the alanine-substituted form of membranedisplayed somatostatin at $\mathrm{Cys}^{14}$ was also constructed. Figure $3 \mathrm{C}$ shows that the strain harboring pYS21-sst (C14A) significantly lost its activity, suggesting that the membrane-displayed somatostatin had a cyclic structure with a disulfide bond between $\mathrm{Cys}^{3}$ and $\mathrm{Cys}^{14}$ residues.

From the results of Figure 3A, the signal intensity of each strain depended on the linker length. One of the reasons for this linker length dependence could be the FLAG tag sequence (DYKDDDDK), which possesses a negatively charged sequence. It is possible that this region interacts with the relatively polar amino acids on the outer loop of GPCRs, which could give rise to linker dependency. Considering that the linker dependency observed in Figure 3A influences the robustness of this technology, membrane-displayed somatostatins without a FLAG tag were constructed to overcome this problem. The linker lengths between somatostatin and the membrane-anchoring domain of Yps1p were designed to have 3,20 , and 38 amino acids (pYS-notag3-sst, pYSnotag20-sst, and pYS-notag38-sst, respectively). Regardless of the linker lengths, the membrane-displayed somatostatins without FLAG tag had activity similar to
pYS21-sst (Figure 3D). This indicates that the negatively charged nature or the secondary structure of the FLAG tag region could have resulted in the linker dependency shown in Figure 3A, and that removal of FLAG tag led to the reduction of linker dependency of this system.

\section{Discussion}

We applied our technology for displaying peptide ligands on a plasma membrane to human SSTR2 that was heterologously produced in yeast cells. Functional activation of the pheromone response pathway by the membranedisplayed somatostatin was successfully achieved. In spite of the previous reports of the functional production of rat SSTR2 (Price et al. 1995) and human SSTR5 (Fukuda et al. 2011) in yeast, this is the first report that documents the functional production of human SSTR2 in yeast. While cell wall trapping of autocrine peptides strategy, in which peptide ligands were displayed on yeast cell wall, has recently been reported (Ishii et al. 2012), the percentage of activated cells by the system was lower than that by our system (Hara et al. 2012a). This suggests that plasma membrane is more suitable than cell wall in terms of peptide-ligand display. The higher accessibility of membrane-displayed ligands to GPCRs may be important for efficient activation. Here, we termed "PepDisplay" as a technology for displaying peptide ligands on a plasma membrane for activation of either endogenous or heterologous GPCRs produced in yeast cells.

In order to estimate the signal intensity triggered by the membrane-displayed somatostatin, the signal intensity of strain pYS-notag38-sst was compared with that of exogenously added somatostatin treatment (Figure 2). The signal intensity was found to be $86.6 \%$ of the maximum response of the exogenously added somatostatin treatment (data not shown). Considering that tethered peptide-induced signal activation ranged from 35\% to $100 \%$ of the soluble hormone-induced maximum intensity in a mammalian experimental system (Fortin et al. 2009), the intensity obtained in this study is reasonable. Although the signal intensity can be improved by further scrutiny (e.g., length and amino-acid composition of the linker region, and expression level of the membranedisplayed ligand), the signal intensity obtained by pYSnotag38-sst was sufficient for detection and sorting by fluorescence-activated cell sorter (Hara et al. 2012a).

To the best of our knowledge, the functional display of cyclic peptides on the plasma membrane has not been previously reported although several research groups have reported on membrane-tethered ligands (Chang et al. 2009; Choi et al. 2009; Fortin et al. 2011; Fortin et al. 2009). Cyclic peptides comprise a promising type of scaffold for peptide therapeutics (Katoh et al. 2011), and the linker dependency of PepDisplay was solved by 


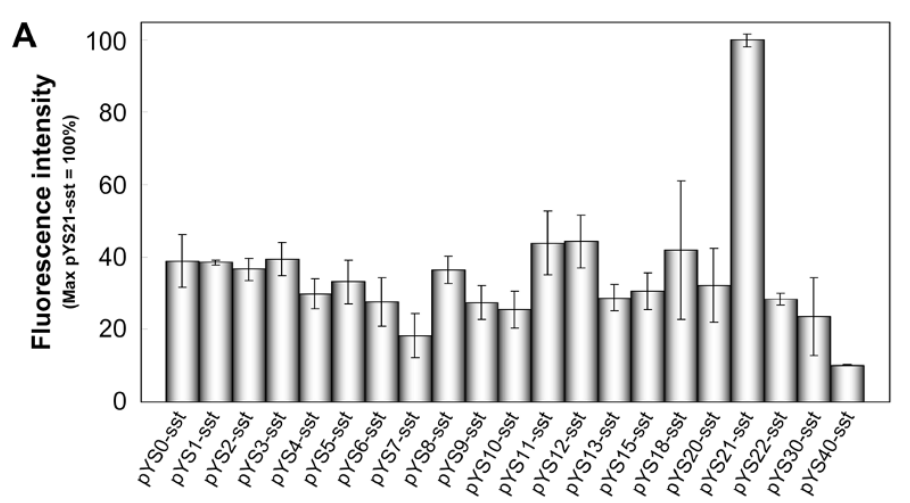

B
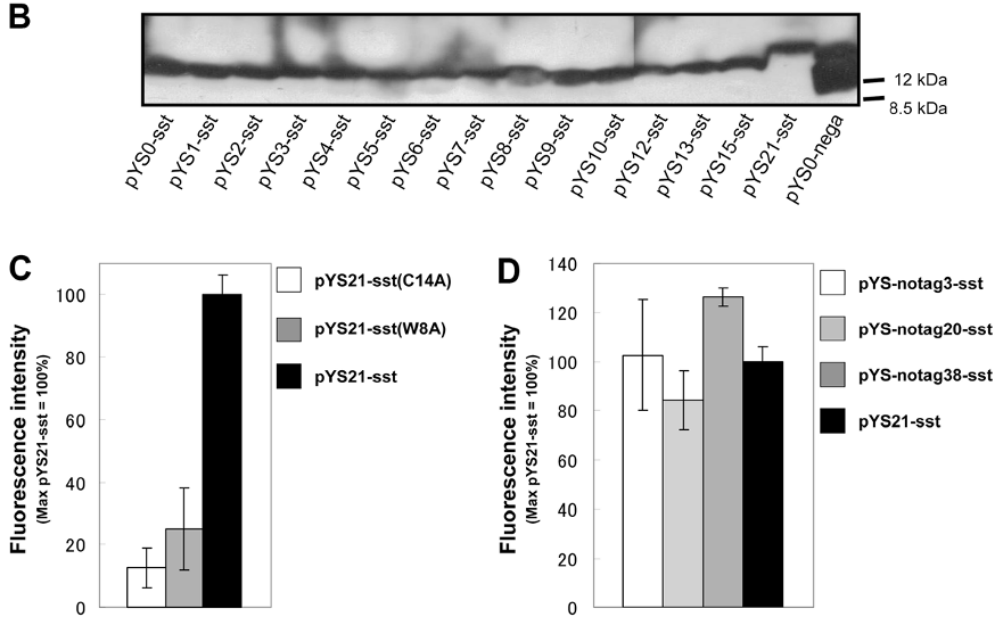

Figure 3 Activation of human somatostatin receptor subtype-2 by membrane-displayed somatostatin. A. Fluorescence intensity of yeast strains transformed with membrane-displayed somatostatin plasmids. A series of yeast strains with one of the membrane-displayed ligand plasmids was assayed in the late-log phase using a 96-well fluorescent plate reader. Yeast cells harboring pYS21-sst emitted strong fluorescence. Each data point represents data (mean \pm standard error of the mean) of three independent experiments. B. Western blot analysis of a series of membrane-displayed somatostatins. Production of membrane-displayed somatostatin on yeast plasma membranes was confirmed by western blot analysis using anti-FLAG tag antibody. The positions of 8.5 and $12 \mathrm{kDa}$ of size markers are indicated by black bars. C. Fluorescence intensity of yeast strains transformed with plasmids for membrane-displayed somatostatin with alanine substitution. Each data point represents data (mean \pm standard error of the mean) of three independent experiments. D. Linker dependency of membrane-displayed somatostatin without FLAG tag upon receptor activation. Fluorescence intensity of yeast strains transformed with plasmids for membrane-displayed somatostatin without FLAG tag. Each data point represents data (mean \pm standard error of the mean) of three independent experiments.

eliminating the FLAG tag; hence, the methodology presented here will provide a new platform for identifying novel peptide ligands for both liganded and orphan mammalian GPCRs.

\section{Abbreviations}

GPCR: G protein-coupled receptor; GPI: Glycosylphosphatidylinositol; EGFP: Enhanced green fluorescent protein; OD: Optical density; SC: Synthetic complete; SSTRs: Somatostatin receptors; SSTR2: Somatostatin receptor subtype-2.

\section{Competing interests}

The authors declare that they have no competing interests.

\section{Acknowledgements}

This study was supported by Research Fellowships from the Japan Society for the Promotion of Science for Young Scientists and, in part, by a Grant-inAid for Scientific Research on Priority Areas "Lifesurveyor" No. 17066002 from the Ministry of Education, Culture, Sports, Science and Technology of Japan through the Single Cell Surveyor Project.
Received: 21 September 2012 Accepted: 23 November 2012

Published: 30 November 2012

\section{References}

Benatuil L, Perez JM, Belk J, Hsieh CM (2010) An improved yeast transformation method for the generation of very large human antibody libraries. Protein Eng Des Sel 23:155-159

Boeke JD, Trueheart J, Natsoulis G, Fink GR (1987) 5-Fluoroorotic acid as a selective agent in yeast molecular genetics. Methods Enzymol 154:164-175 Brachmann CB, Davies A, Cost GJ, Caputo E, Li J, Hieter P, Boeke JD (1998) Designer deletion strains derived from Saccharomyces cerevisiae S288C: a useful set of strains and plasmids for PCR-mediated gene disruption and other applications. Yeast 14:115-132

Brown AJ, Dyos SL, Whiteway MS, White JH, Watson MA, Marzioch M, Clare JJ, Cousens DJ, Paddon C, Plumpton C, and others (2000) Functional coupling of mammalian receptors to the yeast mating pathway using novel yeast/ mammalian $\mathrm{G}$ protein a-subunit chimeras. Yeast 16:11-22

Chang CL, Park JI, Hsu SY (2009) Activation of calcitonin receptor and calcitonin receptor-like receptor by membrane-anchored ligands. J Biol Chem 285:1075-1080 
Chao G, Lau WL, Hackel BJ, Sazinsky SL, Lippow SM, Wittrup KD (2006) Isolating and engineering human antibodies using yeast surface display. Nat Protoc 1:755-768

Choi C, Fortin JP, McCarthy E, Oksman L, Kopin AS, Nitabach MN (2009) Cellular dissection of circadian peptide signals with genetically encoded membranetethered ligands. Curr Biol 19:1167-1175

Dowell SJ, Brown AJ (2002) Yeast assays for G-protein-coupled receptors. Receptors Channels 8:343-352

Eglen RM, Bosse R, Reisine T (2007) Emerging concepts of guanine nucleotidebinding protein-coupled receptor (GPCR) function and implications for high throughput screening. Assay Drug Dev Technol 5:425-451

Fortin JP, Chinnapen D, Beinborn M, Lencer W, Kopin AS (2011) Discovery of dual-action membrane-anchored modulators of incretin receptors. PLoS One 6:e24693

Fortin JP, Zhu Y, Choi C, Beinborn M, Nitabach MN, Kopin AS (2009) Membranetethered ligands are effective probes for exploring class B1 G proteincoupled receptor function. Proc Natl Acad Sci USA 106:8049-8054

Frieman MB, Cormack BP (2004) Multiple sequence signals determine the distribution of glycosylphosphatidylinositol proteins between the plasma membrane and cell wall in Saccharomyces cerevisiae. Microbiology 150:3105-3114

Fukuda N, Ishii J, Kaishima M, Kondo A (2011) Amplification of agonist stimulation of human G-protein-coupled receptor signaling in yeast. Anal Biochem 417:182-187

Gueldener U, Heinisch J, Koehler GJ, Voss D, Hegemann JH (2002) A second set of loxP marker cassettes for Cre-mediated multiple gene knockouts in budding yeast. Nucleic Acids Res 30:e23

Guo M, Aston C, Burchett SA, Dyke C, Fields S, Rajarao SJ, Uetz P, Wang Y, Young K, Dohlman HG (2003) The yeast $G$ protein a subunit Gpa1 transmits a signal through an RNA binding effector protein Scp160. Mol Cell 12:517-524

Hara K, Ono T, Kuroda K, Ueda M (2012a) Membrane-displayed peptide ligand activates the pheromone response pathway in Saccharomyces cerevisiae. J Biochem 151:551-557

Hara K, Inada Y, Ono T, Kuroda K, Yasuda-Kamatani Y, Ishiguro M, Tanaka T, Misaka T, Abe K, Ueda M (2012b) Chimeric yeast G-protein a subunit harboring a 37-residue C-terminal gustducin-specific sequence is functional in Saccharomyces cerevisiae. Biosci Biotechnol Biochem 76:512-516

Hopkins AL, Groom CR (2002) The druggable genome. Nat Rev Drug Discov $1: 727-730$

Ishii J, Yoshimoto N, Tatematsu K, Kuroda S, Ogino C, Fukuda H, Kondo A (2012) Cell wall trapping of autocrine peptides for human G-protein-coupled receptors on the yeast cell surface. PLoS One 7:e37136

Ito K, Sugawara T, Shiroishi M, Tokuda N, Kurokawa A, Misaka T, Makyio H, YurugiKobayashi T, Shimamura T, Nomura N, and others (2008) Advanced method for high-throughput expression of mutated eukaryotic membrane proteins in Saccharomyces cerevisiae. Biochem Biophys Res Commun 371:841-845

Jacoby E, Bouhelal R, Gerspacher M, Seuwen K (2006) The 7 TM G-proteincoupled receptor target family. ChemMedChem 1:761-782

Katoh T, Goto Y, Reza MS, Suga H (2011) Ribosomal synthesis of backbone macrocyclic peptides. Chem Commun (Camb) 47:9946-9958

Kuroda K, Matsui K, Higuchi S, Kotaka A, Sahara H, Hata Y, Ueda M (2009) Enhancement of display efficiency in yeast display system by vector engineering and gene disruption. Appl Microbiol Biotechnol 82:713-719

Ladds G, Goddard A, Davey J (2005) Functional analysis of heterologous GPCR signalling pathways in yeast. Trends Biotechnol 23:367-373

Li B, Scarselli M, Knudsen CD, Kim SK, Jacobson KA, McMillin SM, Wess J (2007) Rapid identification of functionally critical amino acids in a $\mathrm{G}$ proteincoupled receptor. Nat Methods 4:169-174

Low MJ (2004) Clinical endocrinology and metabolism. The somatostatin neuroendocrine system: physiology and clinical relevance in gastrointestinal and pancreatic disorders. Best Pract Res Clin Endocrinol Metab 18:607-622

Mumberg D, Muller R, Funk M (1995) Yeast vectors for the controlled expression of heterologous proteins in different genetic backgrounds. Gene 156:119-122

Patel YC (1999) Somatostatin and its receptor family. Front Neuroendocrinol 20:157-198

Price LA, Kajkowski EM, Hadcock JR, Ozenberger BA, Pausch MH (1995) Functional coupling of a mammalian somatostatin receptor to the yeast pheromone response pathway. Mol Cell Biol 15:6188-6195
Rosenthal LE, Yamashiro DJ, Rivier J, Vale W, Brown M, Dharmsathaphorn K (1983) Structure-activity relationships of somatostatin analogs in the rabbit ileum and the rat colon. J Clin Invest 71:840-849

Siehler S (2008) Cell-based assays in GPCR drug discovery. Biotechnol J 3:471-483

Thomas BJ, Rothstein R (1989) Elevated recombination rates in transcriptionally active DNA. Cell 56:619-630

Thomsen W, Frazer J, Unett D (2005) Functional assays for screening GPCR targets. Curr Opin Biotechnol 16:655-665

Ueda M (2004) Future direction of molecular display by yeast-cell surface engineering. J Mol Catal B: Enzym 28:139-143

Ueda M, Tanaka A (2000) Genetic immobilization of proteins on the yeast cell surface. Biotechnol Adv 18:121-140

Vale W, Rivier J, Ling N, Brown M (1978) Biologic and immunologic activities and applications of somatostatin analogs. Metabolism 27:1391-1401

Weckbecker G, Lewis I, Albert R, Schmid HA, Hoyer D, Bruns C (2003) Opportunities in somatostatin research: biological, chemical and therapeutic aspects. Nat Rev Drug Discov 2:999-1017

Xiao SH, Reagan JD, Lee PH, Fu A, Schwandner R, Zhao X, Knop J, Beckmann H, Young SW (2008) High throughput screening for orphan and liganded GPCRs. Comb Chem High Throughput Screen 11:195-215

Xue C, Hsueh YP, Heitman J (2008) Magnificent seven: roles of G protein-coupled receptors in extracellular sensing in fungi. FEMS Microbiol Rev 32:1010-1032

doi:10.1186/2191-0855-2-63

Cite this article as: Hara et al:: Membrane-displayed somatostatin activates somatostatin receptor subtype- 2 heterologously produced in Saccharomyces cerevisiae. AMB Express 2012 2:63.

\section{Submit your manuscript to a SpringerOpen ${ }^{\circ}$ journal and benefit from:}

- Convenient online submission

Rigorous peer review

- Immediate publication on acceptance

- Open access: articles freely available online

- High visibility within the field

- Retaining the copyright to your article

Submit your next manuscript at $>$ springeropen.com 